\title{
Correlación del IMC con la maduración ósea de vértebras cervicales y edad dental en niños y adolescentes
}

\section{Correlation of BMI with bone maturation of cervical vertebrae and dental age in children and adolescents}

\author{
Jessica Gabriela Laura Cahuana ${ }^{1 \mathrm{a}}$
}

iD https://orcid.org/0000-0003-3446-1801

${ }^{1}$ Investigador independiente.

${ }^{2}$ Maestro en Odontoestomatología, Especialista en Rehabilitación Oral.

\begin{abstract}
Resumen
Objetivo: Determinar la correlación entre el IMC y la maduración ósea de vértebras cervicales y edad dental de pacientes niños y adolescentes atendidos en la Clínica Odontológica de la Universidad Nacional Jorge Basadre Grohmann (2011- 2016). Materiales y métodos: El estudio es de tipo retrospectivo, documental y correlacional. La técnica usada fue la revisión documental de 98 historias clínicas. Los datos obtenidos fueron registrados en la ficha de recolección de datos correspondiente a los estadios de maduración ósea de Hassel y Farman, estadios de calcificación dental, según Demirjian, y las tablas de valoración nutricional antropométrica - IMC; según sexo, peso, talla y edad cronológica. Resultados: Los casos de normopeso son más altos en el sexo femenino (68.25\%); los casos de obesidad son más altos en el sexo masculino (52.9\%). Según el análisis estadístico del IMC y los estadios de maduración ósea evaluados en vértebras cervicales, el 100\% de pacientes que se encuentran en la quinta etapa de maduración ósea son obesos. Existen tendencias notorias de normopeso en las primeras etapas de maduración ósea, y obesidad, en la última etapa de maduración ósea evaluada. Respecto al análisis estadístico del IMC y edad dental, se determinó que actúan en forma independiente, dado que se presentan porcentajes similares de índice de masa corporal en todas las edades dentales. Conclusión: Se concluyó que existe una tendencia al adelantamiento de la aparición de los estadios de maduración ósea, hacia el V estadio de maduración, en pacientes con edades cronológicas comprendidas entre los 13 años y 2 meses hasta los 13 años y 8 meses, con obesidad, y principalmente del sexo masculino.
\end{abstract}

Palabras clave: Índice de masa corporal, calcificación de dientes, desarrollo óseo.

\begin{abstract}
Aim: To determine the correlation between the BMI and the bone maturation of the cervical vertebrae and dental age of children and adolescent patients attended at the Universidad Nacional Jorge Basadre Grohmann dental clinic (2011-2016). Materials and methods: The study is retrospective, documentary and correlational. The technique used was the documentary review of 98 medical records, the data obtained were recorded in the data collection sheet corresponding to the Hassel and Farman bone maturation stages, dental calcification stages according to Demirjian and the anthropometric nutritional assessment tables - BMI according to sex, weight, height and chronological age. Results: Normal weight cases are higher in women (68.25\%), obesity cases are higher in men (52.9\%). According to the statistical analysis of BMI and bone maturation stages evaluated in cervical vertebrae; $100 \%$ of patients who are in the fifth stage of bone maturation are obese. There are notable trends in normal weight in the early stages of bone maturation and obesity in the last stage of bone maturation evaluated. Regarding the statistical analysis of BMI and dental age, it was determined that they act independently, since similar percentages of body mass index are presented in all dental ages. Conclusion: It was concluded that there is a tendency for the appearance of bone maturation stages to advance towards the $\mathrm{V}$ stage of maturation, in patients with chronological ages between 13 years 2 months to 13 years 8 months, with obesity and mainly male.
\end{abstract}

Key words: Body mass index, tooth calcification, bone development. 


\section{Introducción}

Los diferentes ritmos de crecimiento y maduración en el ser humano son el resultado de la interrelación genéticoambiental (1). Cada individuo presenta su propio ritmo de maduración, con su respectivo pico de crecimiento. Así, el tercio medio de la cara y la mandíbula tienden a seguir una curva de crecimiento hasta los 10 años aproximadamente, con un potencial de crecimiento remanente hacia los 20 años. Para la estimación de la edad y maduración se utiliza un conjunto de características orientadoras de la edad, teniendo en cuenta el estado de formación y consolidación del tejido óseo y dental $(2,3)$.

Es importante conocer las estadísticas de los problemas nutricionales por deficiencia, así como aquellos datos resultantes de la sobrealimentación. Según la Encuesta Demográfica y de Salud Familiar - ENDES realizada por el Ministerio de Salud (MINSA), actualmente, en el Perú, el $60 \%$ de la población peruana padece de exceso de peso; las mujeres con una proporción mayor $(63.1 \%)$ a los hombres (56.8 \%). Tacna es la región con mayor población con sobrepeso (40.9\%), luego se ubica la Provincia Constitucional del Callao (39.2\%), La Libertad y Piura (con $38.9 \%$ para cada caso), así como Moquegua y la Región Lima (con 38.8\% para cada caso). Los factores nutricionales influyen en los eventos biológicos relacionados con el crecimiento y desarrollo puberal; cada individuo presenta su propio ritmo de maduración con su respectivo pico de crecimiento, y es en esta etapa del desarrollo donde estas diferencias alcanzan su grado más evidente (4-6).

Esto juega un rol importante en la odontología, especialmente en el área de la ortodoncia y ortopedia. Resulta crucial determinar la maduración esqueletal y el potencial de crecimiento durante la preadolescencia y adolescencia, para alcanzar un diagnóstico y plan de tratamiento correcto. Dentro de los métodos para evaluación de los indicadores de maduración esqueletal y crecimiento están los métodos de análisis de vértebras cervicales observadas en radiografías cefalométricas; la confiabilidad de este tipo de evaluación cérvicovertebral como indicador de crecimiento es de gran importancia, debido a que es independiente del grado de retraso o adelanto con respecto a la edad cronológica, adicionalmente estos métodos reducirían la exposición de rayos $X$, ya que utiliza radiografías tomadas antes de iniciar un tratamiento $(7,8)$.

El desarrollo dental tiene dos aspectos: la formación de las coronas y raíces, y la erupción del diente. De los dos, la formación dental es la más resistente a las influencias ambientales. Existen métodos sobre la base de tablas diseñadas para la predicción de la edad, basándose en estadios de desarrollo, y que son apropiadas cuando se quiere estimar la edad dental de un paciente (8). Según Hilgers et al. (13), los niños con sobrepeso u obesidad podrían acelerar el desarrollo dental; así, el desarrollo dental acelerado en niños obesos es una variable importante a tener en cuenta en la planificación del tratamiento de odontología y ortodoncia pediátrica donde el estadio de desarrollo es crucial.

Se debe considerar la importancia de la evaluación de características individuales de cada paciente; niños, algunos en etapas tempranas de pubertad, y adolescentes con sobrepeso, delgadez o normalidad; en la mejoría de un plan de tratamiento y optimización de resultados. Comprender estos eventos facilitaría el diagnóstico y la terapia ortopédica o quirúrgica para estimular o redireccionar el crecimiento craneofacial $(9,10)$.

El propósito del presente estudio es determinar la correlación entre el IMC y la maduración ósea de vértebras cervicales y edad dental de pacientes niños y adolescentes, atendidos en la Clínica Odontológica de la Universidad Nacional Jorge Basadre Grohmann.

\section{Materiales y métodos}

Este trabajo de investigación es de tipo retrospectivo, documental y correlacional. El universo está constituido por 127 pacientes que acudieron al Área de Ortodoncia y Ortopedia Maxilar (2011-2016), aplicando la fórmula estadística de Cox, y los criterios de inclusión y exclusión; la muestra quedó conformada por 98 historias clínicas con sus respectivas radiografías cefalométricas y panorámicas. Se realizó la revisión documental para recoger los datos necesarios en la ficha de recolección de datos correspondiente al registro de los estadios de maduración ósea de Hassel y Farman (evaluado en radiografías cefalométricas), los estadios de calcificación dental, según Demirjian (evaluados en radiografías panorámicas) y las tablas de valoración nutricional antropométrica, según sexo, talla, peso y edad cronológica obtenida de las historias clínicas. Los datos recolectados fueron trasladados a la base de datos en el programa estadístico SPSS. Se realizó una prueba piloto incluyente con una muestra del $5 \%$ de cada grupo. Para el plan de procesamiento de datos se utilizó un procesamiento computarizado a través del paquete informático SPSS (versión 17). La información obtenida de las unidades de estudio, respecto a las variables a través de los instrumentos, fue ordenada en una matriz de sistematización. Para el plan de análisis de los datos se utilizaron métodos descriptivos generales y analíticos, con un método descriptivo de frecuencias y porcentajes, y ji al cuadrado como medio analítico. 


\section{Resultados}

Tabla 1. Pacientes investigados en la Clínica Odontológica de la Universidad Nacional Jorge Basadre Grohmann. Relación del índice de masa corporal y sexoEn la tabla1, los casos de normopeso son más altos en el sexo femenino; los

\begin{tabular}{|c|c|c|c|c|c|}
\hline & & & \multicolumn{2}{|c|}{ Sexo } & \multirow[b]{2}{*}{ Total } \\
\hline & & & Femenino & Masculino & \\
\hline \multirow[t]{6}{*}{$\mathrm{IMC}$} & \multirow[t]{2}{*}{ Delgadez } & Número & 1 & 0 & 1 \\
\hline & & Porcentaje & $1.7 \%$ & $0.0 \%$ & $1.0 \%$ \\
\hline & \multirow[t]{2}{*}{ Normal } & Número & 43 & 20 & 63 \\
\hline & & Porcentaje & $71.7 \%$ & $52.6 \%$ & $64.3 \%$ \\
\hline & \multirow[t]{2}{*}{ Obesidad } & Número & 16 & 18 & 34 \\
\hline & & Porcentaje & $26.7 \%$ & $47.4 \%$ & $34.7 \%$ \\
\hline \multirow{2}{*}{\multicolumn{2}{|c|}{ Total }} & Número & 60 & 38 & 98 \\
\hline & & Porcentaje & $100.0 \%$ & $100.0 \%$ & $100.0 \%$ \\
\hline
\end{tabular}

Fuente: Matriz de datos.

casos de obesidad son más altos en el sexo masculino. La prueba estadística de ji al cuadrado nos indica que existe dependencias significativas en el comportamiento de estas dos variables; en el sexo femenino la tendencia es hacia la normalidad en el IMC, mientras que en el sexo masculino la tendencia a la obesidad es alta.

Tabla 2. Pacientes investigados en la Clínica Odontológica de la Universidad Nacional Jorge Basadre Grohmann. Relación del índice de masa corporal y maduración ósea, según el método de Hassel y Farman..

\begin{tabular}{|c|c|c|c|c|c|c|c|c|}
\hline & & & \multicolumn{5}{|c|}{ Maduración ósea -Método de Hasssel y Farman } & \multirow[b]{2}{*}{ Total } \\
\hline & & & I & II & III & IV & $\mathbf{V}$ & \\
\hline \multirow[t]{6}{*}{ IMC } & \multirow[t]{2}{*}{ Delgadez } & Número & 1 & 0 & 0 & 0 & 0 & 1 \\
\hline & & Porcentaje & $3.1 \%$ & $0.0 \%$ & $0.0 \%$ & $0.0 \%$ & $0.0 \%$ & $1.0 \%$ \\
\hline & \multirow[t]{2}{*}{ Normal } & Número & 23 & 14 & 14 & 12 & 0 & 63 \\
\hline & & Porcentaje & $71.9 \%$ & $60.9 \%$ & $50.0 \%$ & $92.3 \%$ & $0.0 \%$ & $64.3 \%$ \\
\hline & \multirow[t]{2}{*}{ Obesidad } & Número & 8 & 9 & 14 & 1 & 2 & 34 \\
\hline & & Porcentaje & $25.0 \%$ & $39.1 \%$ & $50.0 \%$ & $7.7 \%$ & $100.0 \%$ & $34.7 \%$ \\
\hline \multirow{2}{*}{\multicolumn{2}{|c|}{ Total }} & Número & 32 & 23 & 28 & 13 & 2 & 98 \\
\hline & & Porcentaje & $100.0 \%$ & $100.0 \%$ & $100.0 \%$ & $100.0 \%$ & $100.0 \%$ & $100.0 \%$ \\
\hline
\end{tabular}

Fuente: Matriz de datos.

En la tabla 2 se evidencia que, del total de pacientes, el 1\% presenta delgadez; el 64.3\% presenta un IMC normal y el $34.7 \%$ presenta obesidad. El 92.3\% de pacientes, en la cuarta etapa de maduración ósea, se encuentra con un IMC normal. El 100\% de pacientes que se encuentran en la quinta etapa de maduración ósea son obesos. Existen tendencias notorias de normopeso en las primeras etapas de maduración ósea, y obesidad, en la última etapa de maduración ósea evaluada (V). Al realizar la prueba de ji al cuadrado, se encuentra que no existe dependencia, por lo tanto, ambas variables actúan en forma independiente. 
Tabla 3. Pacientes investigados en la Clínica Odontológica de la Universidad Nacional Jorge Basadre Grohmann. Relación del índice de masa corporal y edad dental, según el método de Demirjian.

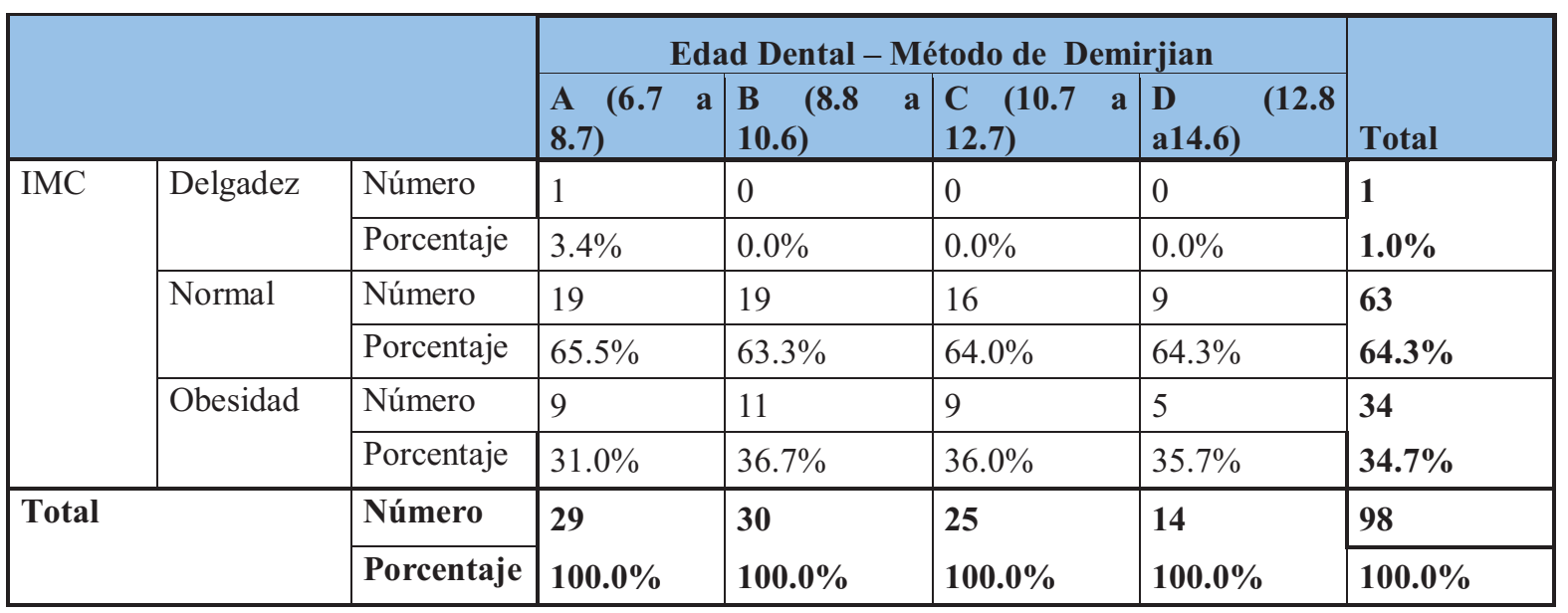

Fuente: Matriz de datos.

En la tabla 3, dentro de los cuatro estadios de la edad dental hallados, se presentan porcentajes similares para el índice de masa corporal en todas las edades dentales. El análisis estadístico de ji al cuadrado nos indica que ambos factores (IMC, edad dental) actúan en forma independiente.

\section{Discusión}

Se considera como un problema de salud pública al sobrepeso y a la obesidad en la población infantil (11); ya que estos presentan una alta probabilidad de presentar obesidad en la edad adulta, esto puede desencadenar problemas sistémicos cardiovasculares y endocrinos a futuro (1). El presente estudio encontró dependencia significativa entre el sexo y el índice de masa corporal, existiendo una tendencia a la normalidad en el sexo femenino y una tendencia a la obesidad del sexo masculino.

Los niños obesos presentan niveles altos de leptina, la misma que genera un efecto directo en los centros de crecimiento esqueletal; por lo tanto, podría ejercer un rol importante en la aparición temprana de la pubertad y en la maduración celular (12-14). Shalitin et al. (1) indican que las niñas con sobrepeso tienden a madurar antes que las niñas con nutrición adecuada; además mantienen la hipótesis de que el grado de grasa corporal puede desencadenar eventos neuroendocrinos que llevan a la aparición temprana de la pubertad. En los niños con obesidad también se ven incrementados los niveles de andrógenos suprarrenales, que pueden estar implicados en el crecimiento acelerado (15). Cuando la maduración ósea se ve adelantada, las posibilidades terapéuticas en las áreas de ortopedia y ortodoncia se ven limitadas; entonces, una aparición temprana de los estadios de maduración ósea es un aspecto importante a considerar para la planificación del tratamiento ortodóncico.
En el presente estudio se encontró que el 100\% de pacientes que adelantaron la etapa de maduración ósea, al quinto y último estadio evaluado, presentaban obesidad; esto significa que según este indicador de maduración esqueletal (SMI) de Hassel y Farman, a estos pacientes les falta solo entre un 5 a $10 \%$ para completar su crecimiento. Coincidentemente Valverde et al. (16) indican, en su estudio, que el sobrepeso influye adelantando el momento de aparición de los estadios de maduración esquelética en escolares de 10 a 15 años de edad, principalmente del sexo femenino. Torres et al. (15), de acuerdo a un análisis de los estadios de maduración ósea de Fishman, encontraron que las niñas de 13 a 15 años presentaron un cese de su crecimiento, en comparación con niñas cuya nutrición era adecuada; esto concuerda con numerosos estudios $(1,12,15,17)$. Lozano et al., quienes analizaron la relación entre la altura con el índice de Quetelet (IMC) y la maduración ósea en niños obesos, concluyeron que los niños obesos mostraron un aumento en la altura, en relación con el adelanto de la edad ósea (18). También coincide con el estudio de Camacho N. quien ratificó el adelanto de la maduración ósea en niños obesos (19).

Además, es importante resaltar que, de acuerdo a algunas investigaciones realizadas en el Perú, existe variabilidad en cuanto al pico de crecimiento puberal (20), que según Hidalgo et al. (21), sucede en mujeres a los 11 años y en varones a los 13.2 años. Mientras que Meneses et al. (22) hallaron el pico de crecimiento a los 10.91 años en el sexo femenino y a los 14.23 años en el sexo masculino. Arriola et al. (23) encontraron que este 
pico aparecía en promedio en mujeres a los $12 \pm 1.2$ y en varones a los $14 \pm 0.8$ años de edad.

Respecto al IMC y edad dental evaluados en el presente estudio, se encontró que ambos actúan de forma independiente, a diferencia de Hilgers et al. (13), quienes señalaron que el desarrollo dental se acelera con el aumento de índice de masa corporal, encontrando aceleración en la edad dental en pacientes con sobrepeso y obesidad; y de Espina de Ferreira A et al. (24), quienes mencionan que sí existe un posible efecto de la nutrición sobre la edad dental (9). Asimismo, Martínez (25) menciona que existe relación entre el estado nutricional y la cronología de erupción dental, siendo que, en la población con pacientes prevalentemente obesos, se encontró un adelanto de la erupción dentaria.
Este estudio aporta información relevante acerca de las variables estudiadas, con un número reducido de estudios sobre la influencia del estado nutricional y sobre la maduración ósea y edad dental específicamente, en una población donde prevalece el sobrepeso y la obesidad. Se sugiere realizar estudios a futuro para contrastar resultados.

\section{Conclusión}

Se concluye que existe una tendencia al adelantamiento de la aparición de los estadios de maduración ósea, hacia el $\mathrm{V}$ estadio de maduración en pacientes con edades cronológicas comprendidas entre los 13 años 2 meses hasta los 13 años 8 meses, con obesidad, y principalmente del sexo masculino.

\section{Referencias bibliográficas}

1. Shalitin S, Phillip M. Role of obesity and leptin in the pubertal process and pubertal growth - a review. Int J Obes Relat Metab Disord. 2003;27(8):869-74.

2. Hernández M. Tratado de endocrinología pediátrica y de la adolescencia. Barcelona. Doyma; 2000.p. 63-81.

3. Uribe R, Gonzalo A. Ortodoncia teoría y clínica. 2da. Edición. Medellín. Colombia. Editorial Corporación para investigaciones biológicas. 2013.p. 15-16.

4. Coelho K, Sichieri R. Influencia de la maduración sexual en el índice de masa corporal en adolescentes de bajo nivel socioeconómico de Rio de Janeiro. Rev Chil Nutr. 2002; 29(1)33-9.

5. Akridge M, Hilgers K, Silveira A, Scarfe W, Scheetz J, Kinane D. Chilhood obesity and skeletal maduration assessed with Fishman hand-writs analysis. Am J Orthod and Dentofac Orthop. 2007; 132(2)185-90.

6. Björk A, Helm S. Prediction of the age of maximum pubertal growth in body height. Angle Orthod. 1967; 37:134-43.

7. Moyers RE. Development of the concept of problems in the treatment of dysmorphism of the face from the viewpoint of the orthodontist. Odontostomatol Proodos. 1981;35(1): p.3-12.

8. Lamparsky D. Skeletal age assessment utilizing cervical vertebrae. Pittsbugh: University of Pittsburgh; 1972.
9. Demirjian A, Goldstein H. New systems for dental maturity based on seven and four teeth. Ann Hum Biol. 1976 Sep;3(5): p.411-21.

10. Vellini, F. Ortodoncia - Diagnóstico y Planificación Clínica. Editorial. Actualidades Médico Odontológicas Latinoamérica C.A. Sao Paulo. 2002.

11. Del Águila CM. Obesidad en el niño: factores de riesgo y estrategias para su prevención en Perú. Rev Peru Med Exp. 2017; 34: 113-8.17.

12. Neeley WW; Gonzales DA. Obesity in adolescence: implications in orthodontic treatment. AJODO. 2007; 131(5):581-8.

13. Hilgers KK, Akridge M, Scheetz JP, Kinane DE. Childhood obesity and dental development. Pediatr Dent. 2006; 28(1):1822.

14. Jasik CB, Lustig RH. Adolescent obesity and puberty: the "perfect storm". Ann N Y Acad Sci. 2008; 1135:265-79.

15. Roque GD, Bonilla P, Meneses A. Influencia del sobrepeso corporal en los estadíos de maduración esquelética en púberes de 12 y 13 años de edad en la ciudad de Huancayo. Rev Estomatol Herediana. 2010;20(2):63-8.

16. Valverde AP, Arriola LE. Aparición de los estadios de maduración esquelética en escolares con sobrepeso y nutrición adecuada. Rev Estomatol Herediana. 2011; 21(4):190196.

17. Martins JCR, Sakima T. Considerações sobre a previsão do surto de crescimento puberal. Ortodontia 1977; 10:164-70. 
18. Lozano B, Chueca S. Estudio de la maduración ósea en niños obesos aragoneses de ambos sexos. Facultad de Medicina de la Universidad de Zaragoza.1996.

19. Camacho C, Velasquez J. Maduración ósea en niños y adolescentes con obesidad. Revista venezolana de endocrinología y metabolismo. Rev. Endocrinol. Metab. 2008.v 6 n. 1.

20. Falen J. Obesidad infanto-juvenil: la epidemia del siglo XXI llegó a Perú. Rev Peru Pediatr. 2014; 67: 128-30.

21. Hidalgo C. Determinación de la curva de crecimiento según Fishman en peruanos de $9 \mathrm{a}$ 16 años de edad. Lima: Universidad Peruana Cayetano Heredia; 1999.

22. Meneses A. Influencia de la altitud geográfica y el estado nutricional sobre los indicadores de maduración esquelética según el índice de Fishman en escolares de 8 a 16 años de edad en la ciudad de Cerro de Pasco. Lima: Universidad Peruana Cayetano Heredia; 2002.

23. Arriola LE, Pardo M. Momento de aparición de los estadíos de maduración esquelética según el método de Fishman en niños y adolescentes de una localidad peruana. Congreso Ortodoncia 2011; 18(1):18-23.

24. Espina A, Ferreira J. Empleo de la edad dental y la edad ósea para el cálculo de la edad cronológica con fines forenses, en niños escolares con alteraciones en el estado nutricional. Universidad del Zulia. 2006.

25. Martinez N. Relación entre el estado nutricional y la cronología de la erupción dental en estudiantes escolares de Tacna. Revista Odontológica Basadrina. 2019. Vol. 3 (2) 11-18.
Correspondencia:

cdgabrielaura@hotmail.com
Fecha de recepción : 07 de abril de 2020

Fecha de aceptación : 08 de junio de 2020 